\title{
Análise estrutural e longitudinal sobre capacidades dinâmicas em agências de publicidade e em mídias sociais
}

Silvia Spagnol Simi dos Santos

Doutoranda em Administração pela Universidade do Oeste de Santa Catarina

Professora da Universidade do Oeste de Santa Catarina

https://orcid.org/0000-0003-3897-1673

silvia.simi@unoesc.edu.br

Sérgio Begnini

Doutorando em Administração pela Universidade do Oeste de Santa Catarina

Mestre em Gestão e Desenvolvimento Regional pela Universidade Estadual do Oeste do Paraná sergio.begnini@unoesc.edu.br

https://orcid.org/0000-0002-7030-6107

Carlos Eduardo Carvalho

Doutor em Administração e Turismo pela Universidade do Vale do Itajaí

Professor da Universidade do Oeste de Santa Catarina

carlos.carvalho@unoesc.edu.br

https://orcid.org/0000-0002-7157-0743

Ieda Margarete Oro

Doutora em Ciências Contábeis e Administração da Universidade Regional de Blumenau

Professora da Universidade do Oeste de Santa Catarina

ieda.oro@unoesc.edu.br

https://orcid.org/0000-0002-2239-531X

Editor Científico: José Edson Lara

Organização Comitê Científico

Double Blind Review pelo SEER/OJS

Recebido em 11.02.2019

Aprovado em 09.12.2019

Este trabalho foi licenciado com uma Licença Creative Commons - Atribuição - Não Comercial 3.0 Brasil 


\title{
Resumo:
}

Objetivo: Neste estudo objetivamos analisar a produção científica das publicações sobre capacidades dinâmicas em agências de publicidades e em mídias sociais.

Metodologia/abordagem: Como metodologia, utilizou-se de uma revisão sistemática de artigos publicados entre os anos de 2008 e 2017, com uso de técnicas bibliométricas. No total, foram analisados 244 artigos os quais foram enumerados e quantificados em relação aos principais autores, journals, bases de dados e ano das publicações.

Originalidade/Relevância: Esta revisão de literatura integrativa das capacidades dinâmicas acrescenta à categorização da literatura da VBR pois relaciona as capacidades dinâmicas com as pesquisas de mídias sociais e agências de publicidade, fornecendo orientações favoráveis para pesquisas futuras. O gap teórico de pauta na escassez de literatura sobre o tema pois é surpreendente que a atividade publicitária, que é ancorada em conhecimento e criatividade, não seja um campo explorado pelas investigações da área da Visão Baseada em Recursos (VBR).

Principais resultados: Os achados apontam para uma tendência de publicações qualitativas com foco em mídias sociais e big data, poucas investigações com foco em agências de publicidade e uma ampla gama de estudos relacionados as capacidades dinâmicas com temáticas de desempenho e inovação.

Contribuições teóricas/metodológicas: Surgem gaps teóricos e a necessidade de mais investigações sobre a indústria criativa, seus recursos e capacidades, especialmente pela ascendência das mídias sociais.

Contribuições sociais/para a gestão: Gestores e profissionais de marketing e comunicação devem depositar seus esforços na análise de big data (big data analytics - BDA) o qual é uma fonte rica de informações dos consumidores em seus pontos de contato.

Palavras-chave: Capacidades Dinâmicas. Mídias Sociais. Agências de Publicidade. Bibliometria.

\section{Structural and longitudinal analysis on dynamic capacities in publicity agencies and social media}

\begin{abstract}
:
Objective: In this study, we aim to analyze the scientific production of publications on dynamic capacities in advertising agencies and social media.

Methodology / approach: The methodology used was a systematic review of the literature. The period used was articles published between 2008 and 2017. In total, 244 articles were analyzed which were enumerated and quantified about the main authors, journals, databases and year of publication.

Originality / Relevance: This integrative literature review of the dynamic capabilities adds to the categorization of the RBV literature as it relates dynamic capabilities to social media research and advertising agencies, providing favorable guidance for future research. The theoretical gap in the literature on the subject is surprising because it is surprising that advertising activity, which is anchored in knowledge and creativity, is not a field explored by investigations in the area of Resource-Based View (RBV).

Key Findings: The findings point to a trend toward qualitative publications focusing on social media and big data, few investigations focusing on advertising agencies, and a wide range of studies related to dynamic capabilities with performance and innovation themes.
\end{abstract}


Theoretical / Methodological Contributions: There is a need for further investigation into the creative industry, its resources, and capabilities, especially the rise of social media.

Social I Management Contributions: Managers, marketers, and communications professionals should focus their efforts on big data analytics (BDA) which is a rich source of consumer information at their touchpoints.

Keywords: Dynamic Capabilities. Social Media. Advertising Agency. Bibliometric.

\section{Análisis estructural y longitudinal sobre capacidades dinámicas en agencias de publicidad y en medios sociales}

\section{Resumen:}

Objetivo: En este estúdio, nos propusimos analizar la producción científica de publicaciones sobre capacidades dinámicas en agencias de publicidad y redes sociales. En este estudio, nuestro objetivo es analizar el stock de conocimiento existente sobre capacidades dinámicas en agencias de publicidad y redes sociales.

Metodología/enfoque: Como metodología, utilizamos una revisión sistemática de los artículos publicados entre 2008 y 2017, utilizando técnicas bibliométricas. En total, se analizaron 244 artículos que se enumeraron y cuantificaron en relación con los principales autores, revistas, bases de datos y año de publicación.

Originalidad/relevancia: Esta revisión de literatura de capacidades dinámicas integradoras se suma a la categorización de la literatura de VBR, ya que relaciona las capacidades dinámicas con las agencias de publicidad y de investigación en redes sociales, proporcionando una guía favorable para futuras investigaciones. La brecha teórica en la escasez de literatura sobre el tema es sorprendente porque es sorprendente que la actividad publicitaria, que está anclada en el conocimiento y la creatividad, no sea un campo explorado por las investigaciones en el área de la Vista basada en recursos (VBR).

Hallazgos clave: Los hallazgos apuntan a una tendencia hacia publicaciones cualitativas centradas en las redes sociales y big data, pocas investigaciones centradas en agencias de publicidad y una amplia gama de estudios relacionados con capacidades dinámicas con temas de rendimiento e innovación.

Contribuciones teóricas/metodológicas: Surgen brechas teóricas y la necesidad de una mayor investigación sobre la industria creativa, sus recursos y capacidades, especialmente el surgimiento de las redes sociales.

Contribuciones sociales/de gestión: Los gerentes y los profesionales de marketing y comunicaciones deben centrar sus esfuerzos en el análisis de big data (BDA), que es una rica fuente de información para el consumidor en sus puntos de contacto.

Palabras clave: Capacidades Dinámicas. Redes sociales. Agencias de Publicidad. Bibliometría.

\section{Introdução}


Firmas inseridas em ambientes de rápidas e constantes mudanças desenvolvem capacidades dinâmicas a fim de entender o ambiente mercadológico turbulento e se reconfigurarem para manter e melhorar seu desempenho (Teece, Pisano, \& Schuen, 1997). As agências de publicidade, inseridas na indústria criativa, convivem neste ambiente de elevada turbulência, e, se reinventam por meio de capacidades dinâmicas para gerar e melhorar a vantagem competitiva. As mídias sociais são uma das principais fontes desta turbulência, influenciando o mercado por meio de mudanças comportamentais nos diversos stakeholders organizacionais. Por outro lado, também são fontes de conhecimento e informação que podem ser explorados nesta dinâmica de adaptação e inovação necessária para o alcance da vantagem competitiva.

Por meio das capacidades dinâmicas, as firmas podem identificar, avaliar e entender as fontes e os procedimentos que envolvem a criação e apropriação de riquezas (Teece, Pisano, \& Schuen, 1997). Capacidades dinâmicas constituem-se em estratégias elaboradas por uma firma, para adquirir, criar e alocar recursos necessários parar gerar novas estratégias a fim de criar valor para a organização, e estabelecer fontes de vantagem competitiva sustentável (Eisenhardt \& Martin, 2000).

Considerando que o papel essencial das agências de publicidade é o de aconselhar e assistir ao cliente em relação à sua comunicação com o mercado (Sant'Anna, Rocha Júnior, \& Garcia, 2009), e que as mídias sociais proporcionam formas diferenciadas de atuação e contato entre as firmas e seus stakeholders (Lam, Yeung, \& Cheng, 2016), as agências que trabalham a partir das mídias sociais, tem a possibilidade acumular conhecimento e aprendizado comercial, além de compartilhar experiências (Nguyen, Xiaoyu, Melewar, \& Chen, 2015). Assim, conhecer a produção científica na intersecção entre estas áreas é relevante para área da administração, pois pode revelar importantes insights para pesquisadores e gestores do campo.

Na última década, houve um número crescente de trabalhos acadêmicos que buscaram aplicar, medir, operacionalizar e reconceituar as capacidades dinâmicas. Tal realidade aponta para a riqueza que estes temas possuem em termos de pesquisa científica, bem como, para os avanços necessários e possíveis. As pesquisas sobre capacidades dinâmicas iniciaram a partir de Teece et al., (1997) e a relação destas com as mídias sociais e agências de publicidade tem crescido a partir de 2008. Assim, a questão de pesquisa é, qual o estoque de conhecimento sobre capacidades dinâmicas em agências de publicidade em mídias sociais? 
Para responder tal pergunta abrangente, o objetivo deste estudo foi analisar a produção científica das publicações sobre capacidades dinâmicas em agências de publicidades e em mídias sociais. Esta investigação faz sentido ao mesmo tempo em que evoca direcionamentos futuros para estudos das próprias empresas de social mídia ou publicidade, pois elas também necessitam ser dinâmicas para conquistarem espaço no mercado competitivo e turbulento da comunicação.

Para organizar, classificar e sistematizar o conhecimento, este trabalho de análise sistemática com propriedades analíticas e bibliométricas focamos em artigos científicos, publicados no período de 2008 a 2017 nas bases Emerald, Ebsco, Spell, Scielo, Science Direct, Elsevier, Springer US, Blakwell Publishers, SAGE Publications, o que culminou em uma amostra de 244 trabalhos. A escolha do período se deu pela ascensão das redes sociais a partir de 2008.

Para identificar e sistematizar os procedimentos técnicos e de pesquisa utilizados, quantificamos os principais autores, periódicos, base de dados e ano das publicações. As análises empíricas realizadas, compreenderam um componente estrutural e longitudinal, facilitando a captura de uma retrospectiva de abordagens, também demonstrando como a pesquisa evoluiu. Os resultados indicam que a maior parte das pesquisas publicadas no período, é qualitativa $(70,9 \%)$, seguidos pelas quantitativas $(25 \%)$ e pelas quali-quanti $(4,1 \%)$. O período apresentou crescimento nas publicações de artigos, sendo 2015 com 61, seguido por 2016 com 56. Os periódicos que mais publicações apresentaram foram o Journal of Knowledge Management e o Technological Forecasting and Social Change.

Este estudo contribui para fornecer uma sistematização de conhecimento no contexto das capacidades dinâmicas em agências de publicidade e de mídias sociais. Este estudo bibliométrico contribui ainda ao descrever questões importantes da pesquisa, ao mesmo tempo que pode indicar gaps de pesquisa com capacidades dinâmicas em agências de publicidade e de mídias sociais, possibilitando à academia visualizar pesquisas futuras que aprofundem essa relação. Também contribui ao identificar questões importantes dos últimos dez anos de pesquisa no campo, como periódicos relevantes, autores que pesquisam os temas e ainda ao proceder análise separadamente dos artigos qualitativos, quantitativos e ambas as abordagens. Ainda, entende-se que as identificações realizadas, por meio deste estudo, podem revelar-se cruciais para maior entendimento do campo, sendo útil, não só, mas em especial para estudantes de mestrado e doutorado, que podem ter uma compreensão rápida da literatura existente nos últimos dez anos. O artigo está dividido em quatro partes. Na seção 2 encontra-se o quadro 
teórico a respeito das capacidades dinâmicas, na seção 3 os procedimentos metodológicos, na seção 4 apresentamos a análise dos resultados e, na seção 5, a conclusão e recomendações.

\section{Quadro Teórico}

A partir da década de 1990, quando a internet passou a integrar o dia-a-dia das empresas, provocou transformações nas organizações, impactando nos processos, rotinas e decisões do dia a dia. Esse movimento foi constante e crescente, sendo que nos dias de hoje há total dependência da internet, e das possibilidades por ela proporcionadas. Tal movimento ocasionou mudanças na forma de gestão e no relacionamento das empresas com seus fornecedores, nos processos de produção, na relação com outras empresas, na forma de buscar financiamentos e nas ações avaliativas em mercados financeiros (Castells, 2003). A internet passou a ser uma ferramenta decisiva de produtividade, bem como de competitividade das empresas (Castells, 2003) em especial aquelas que trabalham com publicidade e com mídias sociais.

Agências de publicidade vivem em um meio, hoje conhecido como "indústria criativa". Este termo teve origem nos anos 90, na Austrália, desenvolvido pela Creative Industries Taskforce, criada em 1997, no Reino Unido. As indústrias criativas são "aquelas que têm origem na criatividade, capacidade e talento individuais, e que potenciam a criação de riqueza e de empregos através da produção e exploração da propriedade intelectual" (Creative Industries Mapping Document [CIMD], 1998). No contexto do presente artigo, pode-se incluir as áreas: design; publicidade; cinema e vídeo; difusão por rádio, internet e televisão e edição (escrita e publicação).

As agências de publicidade também estão modificando seu modelo tradicional de trabalho que advém da gestão estratégica de contas na busca por analisar a co-criação de valor por meio de parcerias entre empresa e clientes estrategicamente importantes (Storbacka, 2012). O novo ambiente proporcionado pelas mídias sociais está transformando os fluxos internos das agências e, ao mesmo tempo, acarreta dúvidas aos anunciantes que anteriormente tinham assertividade quanto ao investimento nas mídias off-line e agora dividem o montante do investimento entre mídia off-line e online. Assim, agências de publicidade estão se transformando e sua atuação na linguagem online tem se tornado igualitariamente necessária a da mídia off-line. A prática atual envolve cada vez mais conhecimento e não se trata apenas de 
concepção de anúncios e sim de análises dos problemas e das oportunidades do cliente, prestando consultorias especializadas para auxiliar as firmas em demandas específicas.

No contexto da competitividade, a gestão estratégica, como campo que possibilita o estudo das organizações, passou a dedicar maior atenção aos conceitos e ações que, de alguma forma, impactam no desempenho das organizações (Hoskisson, Hitt, Wan, \& Yiu, 1999). Para alcançar vantagem competitiva sustentada, as empresas precisam colocar em prática estratégias que desenvolvam valor (Barney, 1991) e obter recursos que ao mesmo tempo sejam heterogêneos, não imitáveis, não substituíveis e de mobilidade imperfeita (Peteraf, 1993). Uma possibilidade para proporcionar maior vantagem competitiva às organizações, são as capacidades dinâmicas, que dizem respeito a habilidade da emprega de integrar, construir e remodelar tanto competências internas, quanto externas, com o objetivo de adaptar-se em resposta às mudanças ambientais (Teece et al., 1997).

Capacidades Dinâmicas (CD) são as rotinas organizacionais e estratégicas ascendentes, pelas quais os gestores alteram a base dos seus recursos, adquirem e criam recursos, os integram e recombinam para gerar uma nova estratégia de criação de valor (Teece et al., 1997). São elas que estão por trás da criação, evolução e recombinação de outros recursos em novas fontes de vantagem competitiva. É onde os gestores integram, constroem e reconfiguram as competências internas e externas, redirecionando para as mudanças. Além disso, são fonte da vantagem competitiva sustentável (Eisenhardt \& Martin, 2000).

Uma estratégia empresarial possibilita o alcance de vantagem competitiva sustentada através de rotinas organizacionais desempenhadas pelas pessoas (Zollo \& Winter, 2002), e conforme aumenta a incidência dessas rotinas é possível desenvolver competências organizacionais. A competência organizacional acontece nas empresas quando as pessoas passam a ter consciência do resultado gerado pelas suas ações no desempenho geral da organização por meio dos recursos que utilizam (Zollo \& Winter, 2002). Os recursos e as capacidades são centrais para formular estratégias e, consequentemente, obter vantagem competitiva sustentada. Recursos e capacidades constituem-se em bases, para que as empresas possam sustentar sua identidade e estabelecer sua estratégia organizacional (Grant, 1996).

Neste estudo nos propomos a estudar as capacidades dinâmicas em contexto caracterizado por elevada turbulência que é o ambiente das agências de publicidade, sendo as mídias sociais um elemento essencial deste contexto.

\section{Procedimentos Metodológicos}


Um estudo bibliométrico considera o progresso científico realizado em determinado período de tempo, sobre um assunto ou a interligação entre mais de um assunto e depende de um conjunto de dados originais que, tenham sido escritos e publicados, com caráter científico (McCain, 1990). A partir da coleta das publicações, procede-se a análise para identificar questões de interesse dos pesquisadores (Gomes, Barnes, \& Mahmood, 2015).

No campo da estratégia, vários estudos bibliométricos foram desenvolvidos para atender propósitos distintos. Como exemplos, podemos citar o trabalho de Ramos-Rodrigues e RuízNavarro (2004) que abordou questões relativas à estrutura intelectual na gestão estratégica. Nerur, Rasheed \& Natarajan (2008) que buscaram traçar a evolução da estrutura intelectual do campo da estratégia. Cardoso, Silva, Silva \& Corso (2013) que se propuseram a identificar autores clássicos e emergentes no campo da estratégia. Shafique (2013) analisou questões referentes a pesquisa em inovação e indicou possíveis pesquisas futuras. Cardoso, Martins \& Kato (2015) que buscaram mapear a evolução das capacidades dinâmicas, analisando as cocitações. Ribeiro \& Corrêa (2018) que aprofundaram questões referentes aos autores e instituições que publicaram sobre estratégia.

A amostra constituiu-se de artigos científicos publicados em periódicos disponíveis nas bases pesquisadas. As bases selecionadas foram: Emerald, Ebsco, Spell, Scielo, Science Direct, Elsevier, Springer US, Blakwell Publishers, SAGE Publications. Para selecionar a amostra, adotou-se de uma série de procedimentos. O primeiro, foi delimitar o período de abrangência da pesquisa entre 2008 a 2017 (até o mês de junho). Em seguida, definiu-se os termos de busca "Dynamic Capabilities" AND "Social Media" e "Dynamic Capabilities" AND "Advertising Agency". A sistematização considerou os operadores lógicos booleanos e unir os termos de busca, visto o extenso volume de artigos relacionados tanto a capacidades dinâmicas, quanto a agências de publicidade e a mídias sociais.

Utilizamos somente os termos na língua inglesa, pois, os mesmos artigos escritos em português, francês, espanhol, por exemplo, quando publicados em periódicos internacionais, possuem palavras-chave em inglês. Delimitamos a busca de publicações, somente na forma de artigos científicos, sendo descartados os demais. Com essas definições realizamos a pesquisa no título, no resumo e nas palavras-chave das publicações, garantindo que os artigos identificados estavam relacionados à temática. 
Encontramos 285 trabalhos. Contudo, previamente, realizou-se a leitura do título e o resumo e foram eliminados aqueles que não atendiam os critérios estabelecidos ou não eram artigos científicos. Chegamos a amostra de 244 artigos publicados no período de 2008 a 2017. Ressaltamos que em 2017 a pesquisa foi realizada até o mês de junho, quando encerramos as buscas. Os 244 artigos foram importados para o software Mendeley, que auxiliou na organização e na leitura, bem como na exportação dos dados, em planilhas para o software Excel, por intermédio do software JabRef. Com os dados na planilha Excel foram realizadas as análises das medidas quantitativas, de autoria, periódicos, método, temas e abordagens teóricas utilizadas.

Na estrutura da análise quantitativa, constatamos necessidade de profundar as temáticas abordadas no estudo. Os diversos artigos tratam de capacidades dinâmicas, porém associadas a outros temas como, sustentabilidade, cadeias de suprimentos, criatividade organizacional, inovação e tecnologia de informação, mas que se utilizam de palavras-chaves que se referem às mídias sociais. Dessa forma, aprofundou-se a análise e compilou-se as informações dos artigos que realmente continham como objeto central de estudo as agências de comunicação ou as mídias sociais. Assim, dos 244 artigos identificados, resultou em uma amostra 78 artigos específicos que caracterizam a indústria criativa (mídias sociais e agências de publicidade).

\section{Apresentação e Análise dos Dados}

Analisar os periódicos em que os artigos foram publicados é uma forma de buscar compreender o conhecimento construído no campo de pesquisa. Apresentar os periódicos que publicam sobre o tema, revela possíveis preferências de abordagens e assuntos. Além disso, possibilita ampliar o entendimento do contexto das capacidades dinâmicas em agências de publicidade e em mídias sociais. Então, é interessante apresentar os periódicos que estão publicando sobre o tema para identificar as bases do campo. A Tabela 1 contém os periódicos que mais publicaram artigos da amostra inicial (dos 244 artigos). Foram 28 periódicos que publicaram ao menos 2 artigos no período do estudo, que representam aproximadamente $35 \%$ da amostra.

Algumas revistas tendem a publicar diversos assuntos, possuindo escopo mais abrangente, outras buscam ser mais específicas, concentrando-se em algumas áreas, ou mesmo em alguns assuntos. Os periódicos que apresentaram maior número de trabalhos relacionadas às temáticas da pesquisa, foram o Journal of Knowledge Management com 11 publicações e o 
Technological Forecasting and Social Change com 8. O Journal of Business Research e o International Journal of Information Management tem 5 publicações e o Journal of the Academy of Marketing Science tem 4.

\section{Tabela 1}

Periódicos com maior número de publicações

\begin{tabular}{|c|c|c|}
\hline Periódicos & Artigos Publicados & $\%$ \\
\hline Journal of Knowledge Management & 11 & 4,51 \\
\hline Technological Forecasting and Social Change & 8 & 3,28 \\
\hline Journal of Business Research & 5 & 2,05 \\
\hline International Journal of Information Management & 5 & 2,05 \\
\hline Journal of the Academy of Marketing Science & 4 & 1,64 \\
\hline Journal of Management & 3 & 1,23 \\
\hline Information Systems Research & 3 & 1,23 \\
\hline Industrial Marketing Management & 3 & 1,23 \\
\hline Electronic Markets & 3 & 1,23 \\
\hline Electronic journal of Information Systems & 3 & 1,23 \\
\hline Business Process Management Journal & 3 & 1,23 \\
\hline Tourism Management & 2 & 0,82 \\
\hline The Journal of Strategic Information Systems & 2 & 0,82 \\
\hline Management Decision & 2 & 0,82 \\
\hline Long Range Planning & 2 & 0,82 \\
\hline The Journal of Management Development & 2 & 0,82 \\
\hline International Small Bussiness Journal & 2 & 0,82 \\
\hline International Journal of Production Economics & 2 & 0,82 \\
\hline International Journal of E-Business Research & 2 & 0,82 \\
\hline Informations Systems Frontiers & 2 & 0,82 \\
\hline Industrial Management Data Systems & 2 & 0,82 \\
\hline Governmental Information Quarterly & 2 & 0,82 \\
\hline European Management Journal & 2 & 0,82 \\
\hline Entrepreneurship: Theory and Practice & 2 & 0,82 \\
\hline Electronic Journal of Knowledge Management & 2 & 0,82 \\
\hline Education + Training & 2 & 0,82 \\
\hline Decision Support Systems & 2 & 0,82 \\
\hline Business Horizons & 2 & 0,82 \\
\hline Total & 85 & 34,84 \\
\hline
\end{tabular}

Fonte: Dados da pesquisa, 2017.

O Journal of Knowledge Management ${ }^{1}$ é uma publicação revisada por pares dedicada ao intercâmbio de pesquisa acadêmica e informações práticas sobre todos os aspectos do gerenciamento de conhecimento nas organizações. A revista publica pesquisa e estudos de caso originais por acadêmicos, empresariais e governamentais sobre estratégias, ferramentas, técnicas e tecnologias para gerenciamento de conhecimento. $O$ foco desta revista é a identificação de estratégias inovadoras de Gestão do Conhecimento e a aplicação de conceitos teóricos a situações do mundo real. O fator de impacto é 2.053 em 2016. Já a Technological Forecasting and Social Change $e^{2}$ tem fator de impacto de 3.129 e é uma revista de acesso aberto da Elsevier que publica pesquisas revisadas por pares com escopo ligado à metodologia e à 
prática da previsão tecnológica e do futuro. O Journal of Business Research ${ }^{3}$ aplica teoria desenvolvida a partir de pesquisas para situações reais de negócios examinando uma variedade de decisões, processos e atividades de negócios dentro da configuração comercial real. Publicado para executivos, pesquisadores e acadêmicos, o journal auxilia a aplicação de pesquisas empíricas em situações práticas e descobertas teóricas sobre a realidade do mundo dos negócios. O International Journal of Information Management ${ }^{4}$ é uma revista que visa a análise e discussão no campo em desenvolvimento da gestão da informação e o Journal of the Academy of Marketing Science ${ }^{5}$ é dedicado ao estudo e aperfeiçoamento do marketing e serve como um elo entre a pesquisa e a prática acadêmica, publicando artigos baseados em pesquisa no domínio substantivo do marketing.

Quanto a classificação dos periódicos que mais publicaram no guia The Association of Business Schools (ABS), as áreas de assunto mais evidentes são a área da Gestão da Informação (8 periódicos), área da Gestão Geral, Ética e Responsabilidade Social (5 periódicos) e área de Marketing (3 periódicos). As áreas Empreendedorismo e Gestão de Pequenas Empresas, Desenvolvimento da Gestão e Educação, Desenvolvimento da Gestão e Educação e Desenvolvimento da Gestão e Educação tiveram 2 periódicos em cada.

Dos 244 artigos extraídos para análise, percebe-se que 173 apresentavam abordagem qualitativa, enquanto 61 foram classificados como quantitativos, e uma menor parte (10), enquadrou-se em nas duas abordagens qualitativa-quantitativa. Essa realidade mostra que a análise qualitativa está predominando e indica que o campo está em fase de amadurecimento. É notável o crescimento no número de trabalhos, visto que enquanto no ano de 2008 foram publicados 2 artigos, no ano de 2016 foram 56, conforme demostrado na Tabela 2.

\section{Tabela 2}

Relação das publicações por ano

\begin{tabular}{ccc}
\hline Ano & Quantidade publicações & \% da amostra \\
\hline 2008 & 2 & 0,819 \\
2009 & 5 & 2,049 \\
2010 & 5 & 2,049 \\
2011 & 13 & 5,327 \\
2012 & 15 & 6,147 \\
2013 & 21 & 8,606 \\
2014 & 32 & 13,114 \\
2015 & 61 & 25 \\
2016 & 56 & 22,950 \\
2017 & 34 & 13,934 \\
Total & 244 & 100 \\
\end{tabular}

Fonte: os autores (2017). 
Observa-se que as publicações sobre capacidades dinâmicas com foco em mídias sociais e agências de publicidade aparecem em 2008, tendo seu ápice em 2015 com 61 produções, sendo, 30 foram quantitativas e 31 qualitativas. Nota-se que em todo o período da pesquisa, a maior parte dos trabalhos é qualitativo, todavia, se isolar, por exemplo, somente o ano de 2015, há considerável crescimento do número de pesquisas quantitativas. Os achados sugerem um aparente crescimento do campo, tendo atingido o ápice no ano de 2015 com 61 publicações.

\subsection{Análise dos artigos quanto ao método}

Nesta etapa, identificou-se os métodos de pesquisa utilizados quanto ao objeto do estudo. Não se pode ver o método somente como um meio de acesso pois é a perspicácia do pesquisador e a sua reflexão que desvendam o que os fatos verdadeiramente são. O método científico percorre os caminhos da dúvida sistemática, e deve ser positivo e não normativo. Em outras palavras, a pesquisa positiva deve se preocupar com o que é e não com o que se pensa que deve ser. (Cervo \& Bervian, 1983).

A maior parte dos trabalhos da amostra são qualitativos. Nestes, o destaque é o estudo de caso (35 ocorrências), seguidas de 2 estudos com análise do discurso. As duas técnicas de coleta de dados predominantemente utilizadas nas pesquisas qualitativas foram ofocus group e a entrevista. Assim, os achados indicam que o estudo de caso ainda é o método mais utilizado pelos pesquisadores em um estudo qualitativo quando pesquisam capacidades dinâmicas em agências de publicidade e de mídias sociais. Também indicam que os autores estão buscando entender melhor o campo.

\section{Tabela 3}

Procedimentos métodos e procedimentos técnicos utilizados nas pesquisas qualitativas

\begin{tabular}{lcc}
\hline \multicolumn{1}{c}{ Categoria } & Número de estudos & \% do total \\
\hline Estudo de caso & 35 & 24,31 \\
Análise do discurso & 2 & 1,39 \\
\hline
\end{tabular}

Fonte: Dados da pesquisa (2017).

A análise técnicas e ferramentas de pesquisa quantitativa foi realizada tendo como base Hair, Black, Babin, Anderson, \& Tatham, (2009). Os achados demonstram que a ferramenta Modelagem de Equações Estruturais é muito utilizada no campo (28 estudos). Essa técnica permite modelar vários constructos, e é adequada também para estudos exploratórios. A regressão e a correlação são utilizadas, sendo que encontramos 16 e 15 estudos respectivamente. 


\section{Tabela 4}

Procedimentos métodos e procedimentos técnicos utilizados nas pesquisas quantitativa

\begin{tabular}{lcc}
\hline \multicolumn{1}{c}{ Categoria } & Número de estudos & \% do total \\
\hline Modelagem de Equações Estruturais & 28 & 31,82 \\
Regressão Linear Hierárquica & 16 & 18,18 \\
Correlação & 15 & 17,05 \\
\hline
\end{tabular}

Fonte: Dados da pesquisa (2017).

Os ensaios e contribuições teóricas são significativos em nossa amostra (55 estudos). O ensaio teórico é uma forma específica de compreensão da realidade, caracteriza-se pela sua natureza reflexiva e interpretativa. No centro do ensaio está a relação quantitativa versus qualitativa, valorizando aspectos relacionados às mudanças qualitativas que ocorrem nos objetos ou fenômenos analisados pelos ensaístas (Meneghetti, 2011). Já a contribuição teórica teoria têm a missão de desafiar e ampliar o conhecimento existente, e não simplesmente reescrevê-lo (Whetten, 1989) e pode se dar tanto ao se testar uma teoria quanto ao criá-la (Colquitt e Zapata-Phelan, 2007). Os achados demonstram que a quantidade de artigos teórico-empíricos é superior aos teóricos, no entanto, há diversas proposições do campo como teoria, no entanto ainda em fase inicial.

Após a análise dos procedimentos e técnicas utilizadas, passamos a revisar a relação dos autores que publicaram mais que um trabalho, com o tipo de método utilizado. Assim, autores que publicaram mais de um artigo no período foram quantificados e identificamos que a quantidade máxima de publicação foi de 2 artigos. Isso se repetiu com 24 autores conforme ilustra a Tabela 05 a seguir.

\section{Tabela 05}

Relação de autores com mais de uma publicação com ano e método utilizado

\begin{tabular}{lccc}
\hline Autor & Quantidade & Ano & Método utilizado \\
\hline Henfridsson, Ola & 2 & 2010,2010 & Qualitativo \\
Ojo, Adegboyega & 2 & 2016,2016 & Qualitativo \\
Akter, Shahriar & 2 & 2015,2016 & Quantitativo \\
Breslin, John G. & 2 & 2016,2016 & Qualitativo \\
Carayannis, Elias G. & 2 & 2013,2017 & Qualitativo \\
Daspit, Joshua J. & 2 & 2015,2016 & Qualitativo \\
Dubey, Rameshwar & 2 & 2015,2016 & Quantitativo \\
Janssen, Marijn & 2 & 2011,2016 & Qualitativo \\
Lyytinen, Kalle & 2 & 2010,2010 & Qualitativo \\
Martin, Graeme & 2 & 2009,2009 & Qualitativo \\
Olszak, Celina M. & 2 & 2016,2017 & Qualitativo \\
Porwol, Lukasz & 2 & 2016,2018 & Qualitativo \\
Reddington, Martin & 2 & 2009,2009 & Qualitativo \\
Sarasvathy, Saras & 2 & 2014,2016 & Qualitativo \\
Teece, David J. & 2 & 2014,2016 & Qualitativo \\
Thomas, Howard & 2 & 2011,2012 & Qualitativo \\
Wamba, Samuel Fosso & 2015,2016 & Quantitativo \\
Yoo, Youngjin & 2 & 2010,2010 & Qualitativo \\
\hline
\end{tabular}




\begin{tabular}{llll}
\hline Zavattaro, Staci M. & 2 & 2015,2016 & Qualitativo \\
Gunasekaran, Angappa & 2 & 2015,2016 & Quantitativo \\
Llorens-Montes, F. & 2 & 2015,2015 & Quantitativo \\
Benitez-Amado, Jose & 2 & 2015,2015 & Quantitativo \\
Childe, Stephen J. & 2 & 2015,2016 & Quantitativo \\
\hline
\end{tabular}

Fonte: os autores (2017)

Identificamos que esses autores publicaram sempre o mesmo tipo de pesquisa (quantitativo/qualitativo), contudo, em anos diferentes. No geral, o ano de publicação foi próximo exceto Janssen, Marijn que publicou seu primeiro artigo em 2011 e o segundo somente em 2016, 5 anos mais tarde. Quanto aos journals que publicaram artigos desses autores citados, 3 deles publicaram artigos dos mesmos autores e do tipo de pesquisa qualitativa as duas vezes: Information Systems Research, com fator de impacto 2,763, publicação dos EUA, Government Information Quarterly, fator de 4.090, Áustria, e Journal of Knowledge Management, fator de impacto 2.054, Alemanha. Os demais publicaram em journals diferentes.

Os achados apontam para uma tendência. Aos pesquisadores se especializarem em determinado método de pesquisa, o que pode ser fruto de uma mesma linha ou grupo de pesquisa.

\subsection{Análise dos temas abordados}

Percebemos que diversos artigos tratam da análise de capacidades dinâmicas com mídias sociais em especial (apenas 1 artigo retrata as agências de publicidade), no entanto, associadas também a outros temas como, sustentabilidade, cadeias de suprimentos, criatividade organizacional, inovação e tecnologia de informação, ou seja, se utilizam de palavras-chaves que se referem às mídias sociais mas não a utilizaram como temática principal. Dessa forma, a importância em aprofundar a análise e compilar informações dos artigos que realmente continham como objeto central de estudo as agências de comunicação ou mídias sociais foi de grande valia, pois dos 244 artigos encontrados no total, abrandamos para uma amostra de 78 artigos específicos a indústria criativa. Passamos assim, a aprofundar nas temáticas desses estudos os quais são o foco central desta investigação.

Quanto ao ano de publicação dos artigos, percebe-se um aumento significativo no ano de 2015 e 2016, o que revela que o campo específico das mídias sociais e das agências de publicidade enxergou as capacidades dinâmicas como teoria que explica as turbulências desta fatia da indústria criativa a partir de então. 
Tabela 06

Relação das publicações com o ano dos artigos específicos

\begin{tabular}{ccc}
\hline Ano & Quantidade publicações & \% da amostra \\
\hline 2008 & 2 & 2,564 \\
2009 & 3 & 3,845 \\
2010 & 2 & 2,564 \\
2011 & 3 & 3,845 \\
2012 & 2 & 2,564 \\
2013 & 4 & 5,128 \\
2014 & 6 & 7,692 \\
2015 & 23 & 29,487 \\
2016 & 26 & 33,333 \\
2017 & 7 & 8,974 \\
Total & 78 & 100 \\
\hline
\end{tabular}

Fonte: os autores (2017)

Os periódicos que mais publicaram esses artigos específicos foram o Journal of Business Research, 5 artigos, Journal of Knowledge Management, Journal of the Academy of Marketing Science, Technological Forecasting and Social Change e Government Information Quarterly, 4 artigos cada e o Electronic Markets 3 artigos.

\section{Tabela 07}

Relação dos journals que mais publicaram artigos específicos

\begin{tabular}{|c|c|c|}
\hline Periódico & Quantidade de publicações & $\%$ \\
\hline Journal of Business Research & 5 & 15,625 \\
\hline Journal of Knowledge Management & 4 & 12,5 \\
\hline Journal of the Academy of Marketing Science & 4 & 12,5 \\
\hline Technological Forecasting and Social Change & 4 & 12,5 \\
\hline Government Information Quarterly & 3 & 9,375 \\
\hline Electronic Markets & 2 & 6,25 \\
\hline Industrial Marketing Management & 2 & 6,25 \\
\hline Information $\{\backslash \&\}$ Management & 2 & 6,25 \\
\hline International Journal of E-Business Research & 2 & 6,25 \\
\hline International Journal of Hospitality Management & 2 & 6,25 \\
\hline The Journal of Strategic Information Systems & 2 & 6,25 \\
\hline Total & 32 & 100 \\
\hline
\end{tabular}

Fonte: os autores (2017)

Os periódicos Journal of Business Research, Journal of Knowledge Management, Journal of the Academy of Marketing Science, Technological Forecasting and Social Change foram citados anteriormente. O Government Information Quarterly ${ }^{6}$, é uma revista que examina a interseção de política, tecnologia da informação, governo e público e se concentra em como as políticas afetam os fluxos de informação do governo e a disponibilidade de informações do governo; o uso de tecnologia para criar e fornecer serviços governamentais inovadores; o impacto da tecnologia da informação na relação entre os governados e os governantes; e a crescente importância das políticas de informação e tecnologia da informação 
em relação às práticas democráticas. Já o Electronic Markets ${ }^{7}$ é uma revista acadêmica trimestral que abrange diversos aspectos da economia digital. Editado na Universidade de Leipzig e publicado pela Springer, emergiu como um dos principais periódicos científicos que se concentram explicitamente em negócios em rede, possibilitados pela tecnologia da informação ("digitalização").

Quanto aos autores que publicaram mais que um artigo com as temáticas específicas estão detalhados na Tabela 08, sendo que os artigos especificados tem métodos qualitativos. Martin Reddington do Reino Unido, Adegboyega Ojo da Irlanda e Joshua J. Daspit de Mississippi.

\section{Tabela 08}

Relação de autores com mais de uma publicação com ano e journal

\begin{tabular}{|c|c|c|c|c|}
\hline Autores & Periódico & Ano & Tema nas firmas & Objeto de Estudo \\
\hline $\begin{array}{l}\text { Reddington, Martin } \\
\text { Reddington, Martin e }\end{array}$ & Employee Relations & 2009 & Capacitação RH & Mídia social e Web 2.0. \\
\hline $\begin{array}{l}\text { Kneafsey, Mary Beth } \\
\text { Ojo, Adegboyega e }\end{array}$ & $\begin{array}{l}\text { Education + Training } \\
\text { Government Information }\end{array}$ & 2009 & Tecnologia & Web 2.0 \\
\hline Breslin, John G. & $\begin{array}{l}\text { Quarterly } \\
\text { Government }\end{array}$ & 2016 & Governança & E-participação \\
\hline Ojo, Adegboyega & $\begin{array}{l}\text { Information } \\
\text { Quarterly }\end{array}$ & 2016 & Governança & $\begin{array}{l}\text { Software Social para } \\
\text { Mídias }\end{array}$ \\
\hline $\begin{array}{l}\text { Daspit, Joshua J. E } \\
\text { Adams, Frank G. }\end{array}$ & $\begin{array}{l}\text { Tourism Management } \\
\text { Journal of Vacation } \\
\text { Marketing }\end{array}$ & 2015 & $\begin{array}{l}\text { Patrimônio de } \\
\text { marca }\end{array}$ & $\begin{array}{l}\text { Redes Sociais } \\
\text { Marketing de destino }\end{array}$ \\
\hline
\end{tabular}

Fonte: os autores (2017)

Na Tabela 08, percebemos temas de estudo similares e com as datas de publicações muito próximas, o que indica que o estudo teve continuidade ou tratou de um mesmo estudo, muitas vezes sendo dividido em duas partes para publicação. O tema mais evidenciado nas investigações acadêmicas do período pesquisado foi a mídia social, a qual possui 25 artigos (Herschel \& Yermish, 2008; Trainor, Andzulis, Rapp, \& Agnihotri, 2014; Kim, Koh, Cha, \& Lee, 2015; Martin \& Reddington, 2009; Bolat, Kooli, \& Wright, 2016; Fisher, McPhail, You, \& Ash, 2014; Braojos-Gomez, Benitez-Amado, \& Llorens-Montes, 2015; Marolt, Pucihar, \& Zimmermann, 2015; Wang \& Kim, 2017; Ahani, Rahim, \& Nilashi, 2017; Lam, Yeung, \& Cheng, 2016; Heartfield \& Loukas, 2016; Venkatarama \& Das, 2013; Chae, 2015; Jalonen, 2014; He, Zheng, \& Zeng, 2016; Dong \& Wu, 2015; Rayna \& Striukova, 2016; Loukis, Charalabidis, \& Androutsopoulou, 2017; Nguyen, Yu, Melewar, \& Chen, 2015; Harrigan, 
Soutar, Choudhury, \& Lowe, 2015; Hong, Shin, \& Kim, 2016; Teo, Nishant, \& Koh, 2016; Barawi \& Seng, 2013 ; Wieneke \& Lehrer, 2016).

As redes sociais foram o segundo tema mais investigado, com um montante de 14 artigos (Vătămănescu, Dumitriu, Andrei, \& Leovaridis, 2015; Yun \& Gloor, 2015; Palacios-Marqués, Merigo, \& Soto-Acosta, 2015; Bharati, Zhang, \& Chaudhury, 2015; Dwivedi, Weerakkody, \& Janssen, 2011; Meng, Zhang, Zhao, Li, \& Guan, 2016; Porwol, Ojo, \& Breslin, 2016; Pinho \& Prange, 2016; Zavattaro, Daspit, \& Adams, 2015; Magnier-Watanabe, Yoshida, \& Watanabe, 2010; Ferreira \& Du Plessis, 2009; Oparaocha, 2016; Brady, Randa, \& Reyns, 2016; Pogrebnyakov, 2017).

Os demais temas foram o big data em 5 artigos (Comuzzi \& Patel, 2016; Erevelles, Fukawa, \& Swayne, 2015; Gupta \& George, 2016; Wamba, et al., 2017; Braganza, Brooks, Nepelski, Ali, \& Moro, 2017), o comércio eletrônico em 5 artigos (Erevelles, Fukawa, \& Swayne, 2015; Hua, Morosan, \& Franco, 2015; Groesser \& Jovy, 2016; Tan, Guo, Cahalane, \& Cheng, 2016; Braganza, Brooks, Nepelski, Ali, \& Moro, 2017) como demonstra a Figura 1, onde as palavras em maior destaque foram as que tiveram a maior quantidade de estudos.

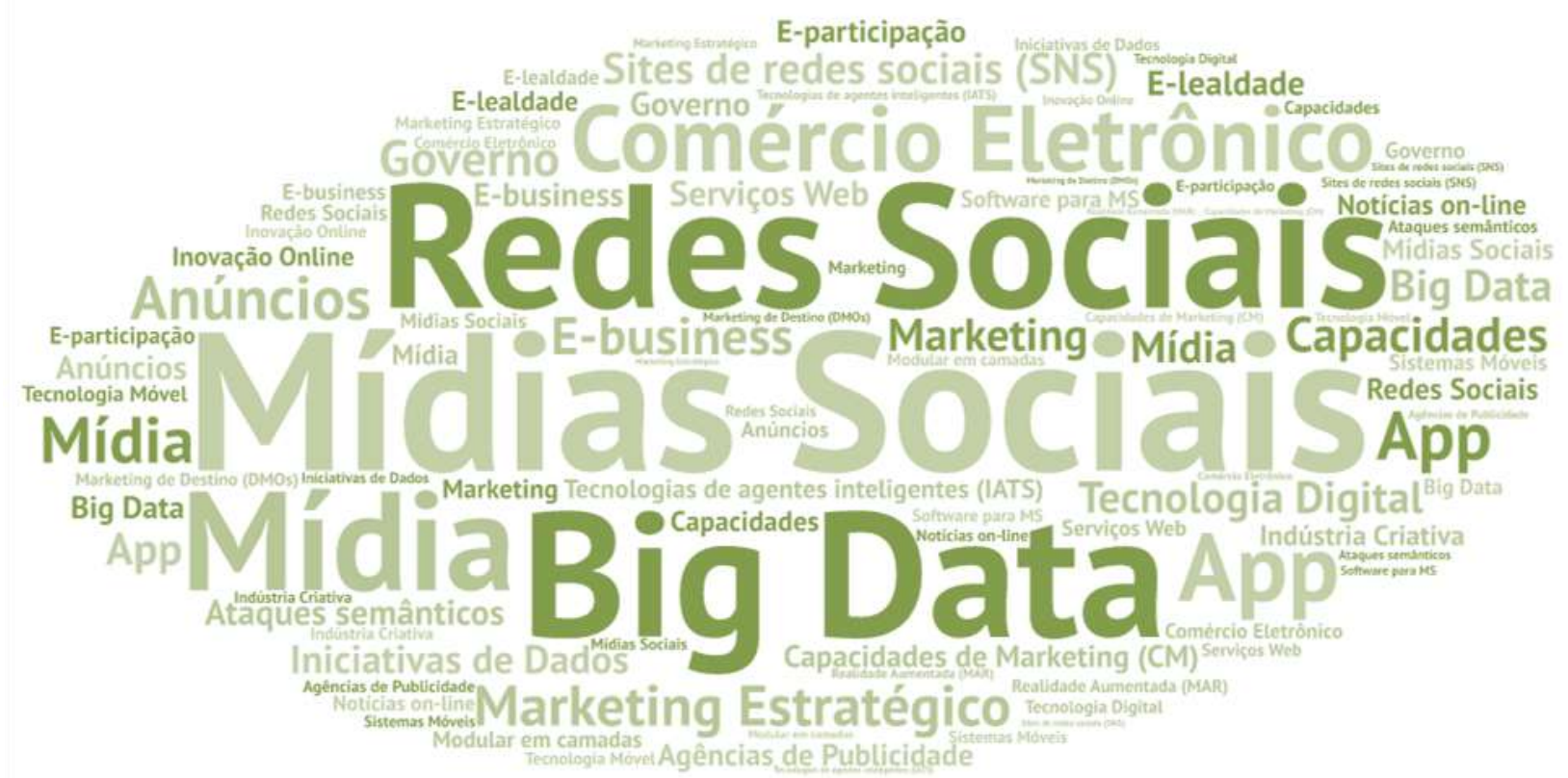

Figura 01. Temas ou objetos de estudo abordados nos artigos Fonte: os autores (2017)

Quanto a dimensão teórica utilizada junto às Capacidades Dinâmicas (CD), as teorias mais adotadas nos artigos são o desempenho com 11 artigos (Gupta \& George, 2016; Wamba, et al., 2017; Hua, Morosan, \& Franco, 2015; Marolt, Pucihar, \& Zimmermann, 2015; Wang \& 
Kim, 2017; Ahani, Rahim, \& Nilashi, 2017; Wilden \& Gudergan, 2015; Morgan, 2012; Yun \& Gloor, 2015; Palacios-Marqués, Merigo, \& Soto-Acosta, 2015; Kamboj \& Rahman, 2015), o marketing com 8 artigos (Wang, Lai, \& Chang, 2016; Wilden \& Gudergan, 2015; Kamboj \& Rahman, 2015; Zavattaro \& Daspit, 2016; Josephson, Johnson, \& Mariadoss, 2016), (Kumar, Dixit, Javalgi, \& Dass, 2016), a inovação com 7 artigos (Kamprath \& Mietzner, 2015; Zavattaro \& Daspit, 2016; Mäkimattila, Saunila, \& Salminen, 2014; Dong \& Wu, 2015; Yoo, Henfridsson, \& Lyytinen, 2010; Nylén \& Holmström, 2015) e a governança em 4 artigos (Dwivedi, Weerakkody, \& Janssen, 2011), (Meng, Zhang, Zhao, Li, \& Guan, 2016; Porwol, Ojo, \& Breslin, 2016).

Outros temas tiveram 3 artigos, a saber: o capital intelectual (MorenoJunior \& Gomes, 2012; Vătămănescu, Dumitriu, Andrei, \& Leovaridis, 2015; Erevelles, Fukawa, \& Swayne, 2015) a competitividade (MorenoJunior \& Gomes, 2012; Josephson, Johnson, \& Mariadoss, 2016; Braojos-Gomez, Benitez-Amado, \& Llorens-Montes, 2015), o gerenciamento (Braganza, Brooks, Nepelski, Ali, \& Moro, 2017; Akama, Cooper, \& Mees, 2016; Trainor, Andzulis, Rapp, \& Agnihotri, 2014) e a internacionalização (Huang, Hu, \& Chen, 2008; Pinho \& Prange, 2016). Todos os demais com 2 ou 1 artigo de cada assunto.

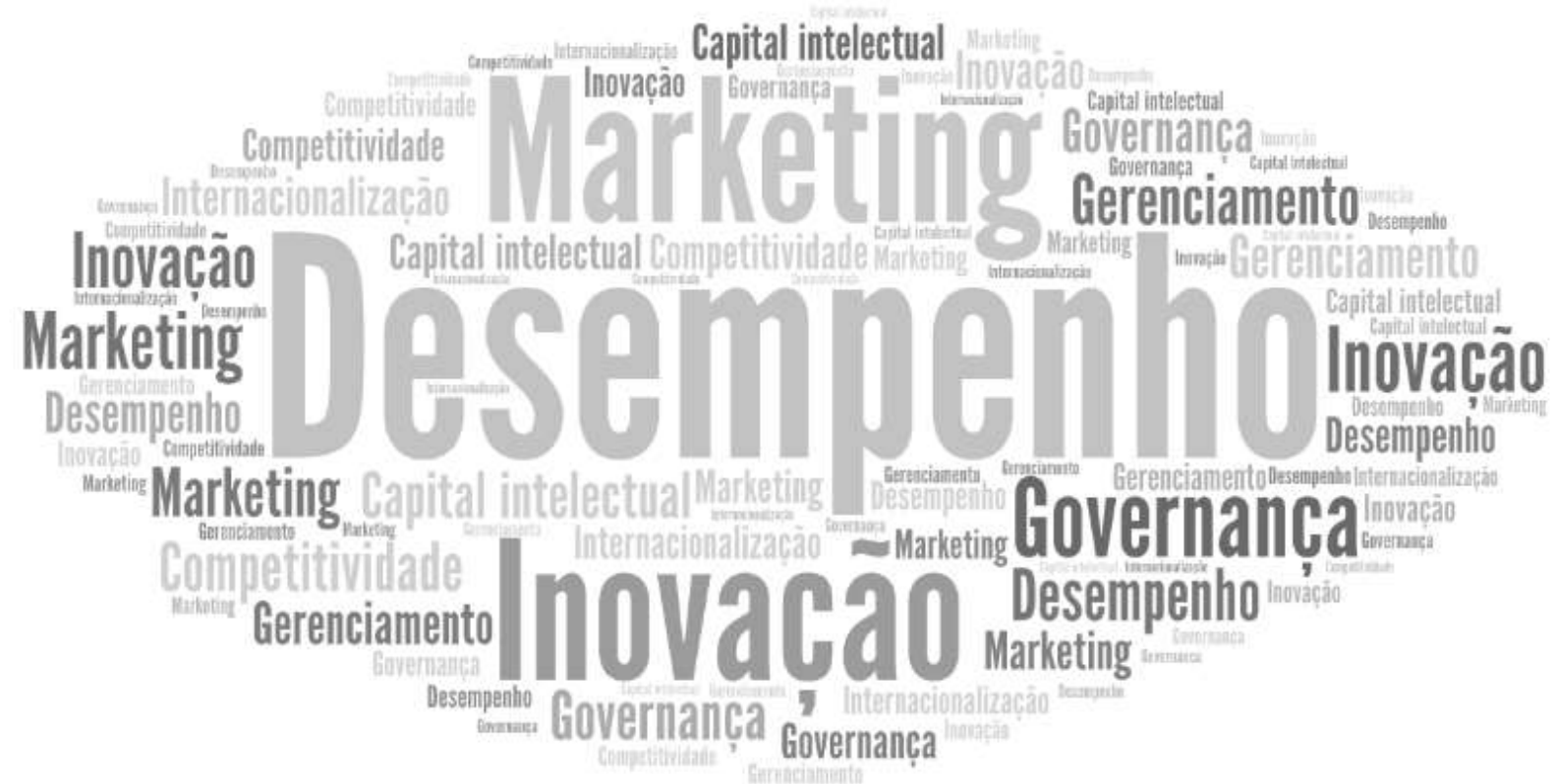

Figura 02. Teorias abordadas junto às $C D$

Fonte: os autores (2017) 
Os achados vislumbram os temas atuais que se referem aos diversos campos das Redes Sociais e fornecem direções futuras de estudos onde as Capacidades Dinâmicas das firmas fomentadas por essa inter-relação é fato consolidado deste ambiente mercadológico. A digitalização do comportamento do consumidor reflete nos investimentos em mídia das marcas e, consequentemente, nos negócios digitais fomentados pelo $e$-commerce, que, com ferramentas como o big data chegam a um público cada vez mais segmentado por meio das métricas fornecidas pelas diversas mídias sociais. Por meio do BDA, os profissionais deste segmento estão obtendo insights de alto valor sobre seus consumidores, concorrentes e o mercado de forma geral.

\section{Conclusões e Recomendações}

As capacidades dinâmicas sustentam as fontes e métodos de criação e a apropriação de como as empresas gerenciam e se comportam em ambientes de rápida mudança tecnológica (Teece et al., 1997). Este trabalho objetivou analisar a produção científica das publicações sobre capacidades dinâmicas em agências de publicidades e em mídias sociais. Foram identificados como principais objetos de estudos as redes sociais, as mídias sociais, o big data e o comércio eletrônico e como principais abordagens teóricas relacionadas as capacidades dinâmicas aquelas preocupadas com a explicação do desempenho, da inovação, da governança, do marketing, do capital intelectual, da competitividade, do gerenciamento e da internacionalização das empresas. Portanto, este artigo contribui para a compreensão da literatura a respeito das $\mathrm{CD}$ com foco nas mídias sociais e em agências de publicidade.

Com base nos resultados, existe uma tendência de publicações qualitativas sobre capacidades dinâmicas com foco em mídias ou redes sociais, e automaticamente a necessidade de mais pesquisas quantitativas sobre esse objeto de estudo pela crescente ascendência das mídias sociais e métricas empregadas neste contexto. Porém, no período mais recente os estudos quantitativos vêm apresentando crescimento significativo. É natural que o desenvolvimento de um campo de pesquisa inicie com estudos teóricos que, primeiramente passam a ser testados empiricamente em estudos exploratórios qualitativos, e que somente após certa consolidação do campo passem a predominar os estudos quantitativos hipotético-dedutivos.

Com o passar dos anos, a medida que o número de pesquisas aumentar, os ensaios teóricos tendem a deixar de ser predominantes. Não identificamos autores proeminentes, o que 
também demonstra o estágio de baixa maturidade do campo. Espera-se que a partir da evolução dos estudos na área, autores mais prolíficos surjam e dominem o campo.

Este trabalho atentou-se para apenas uma pequena parte da Indústria Criativa. Estudos futuros podem focar em outros ramos deste ambiente. Ainda há espaço para contribuições adicionais das $\mathrm{CD}$ em várias áreas relacionadas às mídias digitais. Perguntas futuras de pesquisas a serem desenvolvidas poderão focar na análise dos processos e capacidades dinâmicas das mídias sociais nas empresas. Também sugerimos análises das próprias empresas desse ramo (agências de publicidade, agências de mídia online, setores internos de comunicação das firmas, entre outras), bem como as oportunidades, desafios e obstáculos quando as empresas integram novos conhecimentos trazidos além de seus limites, de diversos parceiros e contextos.

\section{Notas Finais}

${ }^{1}$ Journal of Knowledge Management. (2017). Recuperado de https://www.emeraldinsight.com/journal/jkm

${ }^{2}$ Technological Forecasting and Social Change. (2017). Recuperado de https://www.journals.elsevier.com/technological-forecasting-and-social-change/

${ }^{3}$ Journal of Business Research. (2017). Recuperado de https://www.journals.elsevier.com/journal-of-business-research

${ }^{4}$ International Journal of Information Management. (2017). Recuperado de https://www.journals.elsevier.com/international-journal-of-information-management ${ }^{5}$ Journal of the Academy of Marketing Scienc. (2017). Recuperado de https://www.springer.com/business+\&+management/journal/11747

${ }^{6}$ Government Information Quarterl. (2017). Recuperado de https://www.journals.elsevier.com/government-information-quarterly

${ }^{7}$ Electronic Markets. (2017). Recuperado de http://www.electronicmarkets.org/

\section{Referências}

Ahani, A., Rahim, N. Z., \& Nilashi, M. (October de 2017). Forecasting social CRM adoption in SMEs: A combined SEM-neural network method. Computers in Human Behavior, 75, pp. 560-578.

Akama, Y., Cooper, V., \& Mees, B. (8 de February de 2016). Beyond transmission: An analysis of communication frameworks in Australian bushfire preparedness. International Journal of Disaster Resilience in the Built Environment, 7(1), pp. 63-72.

Barawi, M. H., \& Seng, Y. Y. (2013 ). Evaluation of Resource Creations Accuracy by Using Sentiment Analysis. Procedia - Social and Behavioral Sciences, 97, pp. 522-527

Barney, J. B. (1991). Firm resources and sustained competitive advantage. Journal of management, 17(1), 99-120. 
Bharati, P., Zhang, W., \& Chaudhury, A. (2015). Better knowledge with social media? Exploring the roles of social capital and organizational knowledge management. Journal of Knowledge Management, 19(3), pp. 456-475.

Bolat, E., Kooli, K., \& Wright, L. (2016). Businesses and mobile social media capability. Journal of Business \& Industrial Marketing, 31(8), pp. 71-981.

Brady, P. Q., Randa, R., \& Reyns, B. W. (December de 2016). From WWII to the World Wide Web: A Research Note on Social Changes, Online "Places," and a New Online Activity Ratio for Routine Activity Theory. Journal of Contemporary Criminal Justice, 32(2), pp. 129-147.

Braganza, A., Brooks, L., Nepelski, D., Ali, M., \& Moro, R. (January de 2017). Resource management in big data initiatives: Processes and dynamic capabilities. Journal of Business Research, 70, pp. 328-337.

Braojos-Gomez, J., Benitez-Amado, J., \& Llorens-Montes, F. J. (August de 2015). How do small firms learn to develop a social media competence? International Journal of Information Management, 35(4), pp. 443-458.

Cardoso, A. L. J., Martins, T. S., \& Kato, H. T. (2015). Tendências temáticas em capacidades dinâmicas: um mapeamento do campo por meio de um estudo de co-citações. Revista de Administração e Inovação - RAI, 12(2), 38-59.

Cardoso, A. L. J., Silva, W. V., Silva, E. D., \& Del Corso, J. M. (2013). Análise das publicações mais citadas do Strategic Management Journal no período de 2001 a 2010. Revista Ibero-Americana de Estratégia - RIAE, 12(3) 281-312.

Castells, M. (2003). A galáxia da internet. Reflexões sobre a internet, os negócios e a sociedade. Rio de Janeiro: Jorge Zahar Editor.

Cervo, A., Brevian, P.A. (1983). A metodologia científica. São Paulo: McGraw-Hill.

Chae, B. (. (July de 2015). Insights from hashtag \#supplychain and Twitter Analytics: Considering Twitter and Twitter data for supply chain practice and research. 165, pp. 247-259.

Colquitt, J. A.; Zapata-Phelan, C. P. (2007). Trends in theory building and theory testing: a five-decade study of the academy of management journal. The Academy of Management Journal, 50(6), p. 1281-1303.

Comuzzi, M., \& Patel, A. (September de 2016). How organisations leverage Big Data: a maturity model. Industrial Management \& Data Systems, 116(8), pp. 1468-1492.

Creative Industries Mapping Document. (1998). Creative Industries Mapping Documents. São Paulo. Recuperado de https://assets.publishing.service.gov.uk/government/uploads/system/uploads/attachment_ data/file/193572/Creative_Industries_Mapping_Document_Advertising.pdf.

Dong, J. Q., \& Wu, W. (June de 2015). Business value of social media technologies: Evidence from online user innovation communities. The Journal of Strategic Information Systems, 24(2), pp. 113-117.

Dwivedi, Y. K., Weerakkody, V., \& Janssen, M. (November de 2011). Moving Towards Maturity: Challenges to Successful E-government Implementation and Diffusion. The DATA BASE for Advances in Information Systems, 42(4), pp. 11-22.

Eisenhardt, K. M.; Martin, J. A. (2000). Dynamic Capabilities: what are they? Strategic Management Journal, 21, 1105-1121. 
Erevelles, S., Fukawa, N., \& Swayne, L. (2015). Big Data consumer analytics and the transformation of marketing. Journal of Business Research, 69, pp. 897-904.

Ferreira, A., \& Du Plessis, T. (12 de February de 2009). Effect of online social networking on employee productivity. SA Journal of Information Management, 1.

Fisher, R., McPhail, R., You, E., \& Ash, M. (2014). Using social media to recruit global supply chain managers. International Journal of Physical Distribution \& Logistics Management, 44(8/9), pp. 635-645.

Gomes, E., Barnes, B. R., \& Mahamood, T. (2015). A 22 year review of strategic alliance research in the leading management journals. International Business Review, 25(1), 15-27.

Grant, R. M. (1996). Toward a knowledge-based theory of the firm. Strategic Management Journal, 17, 109-122.

Groesser, S., \& Jovy, N. (February de 2016). Business model analysis using computational modeling: a strategy tool for exploration and decision-making. Journal of Management Control: Zeitschrift für Planung und Unternehmenssteuerung, 27(1), pp. 61-88.

Gupta, M., \& George, J. F. (December de 2016). Toward the development of a big data analytics capability. Information \& Management, 53(8), pp. 1049-1064.

Harrigan, P., Soutar, G., Choudhury, M. M., \& Lowe, M. (February de 2015). Modelling CRM in a social media age . Australasian Marketing Journal (AMJ), 23(1), pp. 27-37.

He, S., Zheng, X., \& Zeng, D. (January de 2016). A model-free scheme for meme ranking in social media. Decision Support Systems, 81, pp. 1-11.

Heartfield, R., \& Loukas, G. (February de 2016). A Taxonomy of Attacks and a Survey of Defence Mechanisms for Semantic Social Engineering Attacks. ACM Computing Surveys (CSUR), 48(3), pp. 1-39.

Herschel, R., \& Yermish, I. (2008). Knowledge Transfer: Revisiting Video. International Journal of Knowledge Management, 4(2), pp. 62-74.

Hong, Y. J., Shin, D., \& Kim, J. H. (September de 2016). High/low reputation companies' dialogic communication activities and semantic networks on Facebook: A comparative study. Technological Forecasting and Social Change, 110, pp. 78-92.

Hoskisson, R., Hitt, M., Wan, W., \& Yiu, D. (1999). Theory and research in strategic management: swings of a pendulum. Journal of Management. 25(3), 417-456.

Hua, N., Morosan, C., \& Franco, A. D. (February de 2015). The other side of technology adoption: Examining the relationships between e-commerce expenses and hotel performance. International Journal of Hospitality Management, 45, pp. 109-120.

Huang, T.-Y., Hu, J.-S., \& Chen, K.-C. (October de 2008). The influence of market and product knowledge resource embeddedness on the international mergers of advertising agencies: The case-study approach. International Business Review, 17(5), pp. 587-599

Jalonen, H. (2014). A framework for dealing with fundamental knowledge problems through social media. VINE Journal of Information and Knowledge Management Systems, 44(4), pp. 558-578.

Josephson, B. W., Johnson, J. L., \& Mariadoss, B. J. (539-554 de 2016). Strategic marketing ambidexterity: antecedents and financial consequences. Journal of the Academy of Marketing Science, 44(4). 
Kamboj, S., \& Rahman, Z. (November de 2015). Marketing capabilities and firm performance: literature review and future research agenda. International Journal of Productivity and Performance Management, 64(8), pp. 041-1067.

Kamprath, M., \& Mietzner, D. (June de 2015). The impact of sectoral changes on individual competences: A reflective scenario-based approach in the creative industries. Technological Forecasting and Social Change, 95, pp. 252-275.

Kim, S., Koh, Y., Cha, J., \& Lee, S. (August de 2015). Effects of social media on firm value for US restaurant companies. International Journal of Hospitality Management, 49, pp. 40-46.

Kumar, V., Dixit, A., Javalgi, R. G., \& Dass, M. (2016). Research Framework, Strategies, And Applications Of Intelligent Agent Technologies (IATs) In Marketing. Journal of the Academy of Marketing Science, 44(1), pp. 24-45.

Lam, H. K., Yeung, A. C., \& Cheng, T. C. (November de 2016). The impact of firms' social media initiatives on operational efficiency and innovativeness. Journal of Operations Management, 47-48, pp. 28-43 .

Loukis, E., Charalabidis, Y., \& Androutsopoulou, A. (January de 2017). Promoting open innovation in the public sector through social media monitoring. Government Information Quarterly, 34(1), pp. 99-109.

Mäkimattila, M., Saunila, M., \& Salminen, J. (January de 2014). Interaction and innovation-reframing innovation activities for a matrix organization . Interdisciplinary Journal of Information, Knowledge and Management, 9, pp. 131-152.

Magnier-Watanabe, R., Yoshida, M., \& Watanabe, T. (October de 2010). Social network productivity in the use of SNS. Journal of Knowledge Management, 14(6), pp. 910-927.

Marolt, M., Pucihar, A., \& Zimmermann, H.-D. (November de 2015). Social CRM adoption and its impact on performance outcomes: a literature review. Organizacija, 48(4), pp. 260-271.

Martin, G., \& Reddington, M. (August de 2009). Reconceptualising absorptive capacity to explain the e-enablement of the HR function (e-HR) in organizations. Employee Relations , 31(5), pp. 515-537.

Meng, Q., Zhang, N., Zhao, X., Li, F., \& Guan, X. (February de 2016). The governance strategies for public emergencies on social media and their effects: a case study based on the microblog data. Electronic Markets, 26(1), pp. 15-29.

MorenoJunior, V. d., \& Gomes, J. C. (s.d.). Benefits and success factors of open-source web services development platforms for small software houses. JISTEM - Journal of Information Systems and Technology Management, 9(8), pp. 585-606.

Morgan, N. A. (January de 2012). Marketing and business performance . Journal of the Academy of Marketing Science, 40(1), pp. 102-119.

Meneghetti, F.K. (2011) O que é um ensaio-teórico? Revista de Administração Contemporânea. 15(2). RAC, Curitiba, pp. 320-332. Recuperado de http://www.scielo.br/scielo.php?script=sci_arttext\&pid=S1415-65552011000200010

McCain, K. (1990). Mapping authors in intellectual space: a technical overview. Journal or the American Society for Information Science, 41(6), 433-443.

Nerur, S. P., Rasheed, A. A., \& Natarajan, V. (2008). The intellectual structure of the strategic management field: an author co-citation analysis. Strategic Management Journal, 29, 319336. 
Nguyen, B., Yu, X., Melewar, T. C., \& Chen, J. (November de 2015). Brand innovation and social media: Knowledge acquisition from social media, market orientation, and the moderating role of social media strategic capability. Industrial Marketing Management, 51, pp. 11-25.

Nylén, D., \& Holmström, J. (January-February de 2015). Digital innovation strategy: A framework for diagnosing and improving digital product and service innovation. Business Horizons, 58(1), pp. 57-67.

Oparaocha, G. O. (2016). Towards building internal social network architecture that drives innovation: a social exchange theory perspective. Journal of Knowledge Management, 20(3).

Palacios-Marqués, D., Merigo, J. M., \& Soto-Acosta, P. (October de 2015). Online social networks as an enabler of innovation in organizations. Management Decision, 53(9), pp. 1906-1920.

Pinho, J. C., \& Prange, C. (April de 2016). The effect of social networks and dynamic internationalization capabilities on international performance. Journal of World Business, 51(3), pp. 391-403 .

Pogrebnyakov, N. (2017). A cost-based explanation of gradual, regional internationalization of multinationals on social networking sites.(RESEARCH ARTICLE). Management International Review, 57(1), pp. 37-64.

Porwol, L., Ojo, A., \& Breslin, J. G. (July de 2016). An ontology for next generation eParticipation initiatives. Government Information Quarterly, 33(3), pp. 583-594.

Porwol, L., Ojo, A., \& G.Breslin, J. (October de 2016). Social Software Infrastructure for e-Participation. Government Information Quarterly, 35(4), pp. 588-598 .

Rayna, T., \& Striukova, L. (25 de April de 2016). 360 Business Model Innovation: Toward an Integrated View of Business Model Innovation An integrated, value-based view of a business model can provide insight into potential areas for business model innovation. Research-Technology Management, 59(3), pp. 21-28.

Tan, F. T., Guo, Z., Cahalane, M., \& Cheng, D. (November de 2016). Developing business analytic capabilities for combating e-commerce identity fraud: A study of Trustev's digital verification solution. Information \& Management, 53(7), pp. 878-891 .

Ribeiro, H. C. M. \& Corrêa, R. (2018). Revista Ibero-Americana de Estratégia: produção acadêmica de 2002 a 2015. Perspectivas em Gestão \& Conhecimento, 8(1), 210-231

Sant'anna, A., Rocha Júnior, I., \& Garcia, L. F. (2009). Propaganda: teoria, técnica e prática. (8a. ed.). São Paulo: Cengage Learning.

Shafique, M. (2013). Thinking inside the box? Intellectual Structure of the knowledge base of Innovation research (1988-2008). Strategic Management Journal, 34(1), 62-93.

Storbacka, K. (2012). Strategic account management programs: alignment of design elements and management practices. Journal of Business \& Industrial Marketing, 27(4), 259-274.

Teece, D., Pisano, G., \& Shuen, A. (1997). Dynamic capabilities and strategic management. Strategic Management Journal, 18(7), 509-534.

Teo, T. S., Nishant, R., \& Koh, P. B. (December de 2016). Do shareholders favor business analytics announcements? The Journal of Strategic Information Systems, 25(4), pp. 259276. 
Trainor, K. J., Andzulis, J. (., Rapp, A., \& Agnihotri, R. (June de 2014). Social media technology usage and customer relationship performance: A capabilities-based examination of social CRM. Journal of Business Research, 67(6), pp. 1201-1208 .

Vătămănescu, E.-M., Dumitriu, D.-L., Andrei, A. G., \& Leovaridis, C. (October de 2015). Networking Intellectual Capital towards Competitiveness: An Insight into the European Higher Education Institutions. . Electronic Journal of Knowledge Management , 13(3), pp. 228-239.

Venkatarama, S., \& Das, R. (2013). The Influence of Corporate Social Media on Firm Level Strategic Decision Making: A Preliminary Exploration. International Journal of E-Business Research, 9(1), pp. 1-20.

Wamba, S. F., Gunasekaran, A., Akter, S., Ren, S. J.-f., Dubey, R., \& Childe, S. J. (2017).

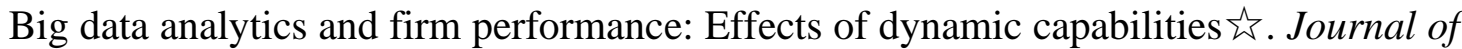
Business Research, 70, pp. 356-365.

Wang, J., Lai, J.-Y., \& Chang, C.-H. (October de 2016). Modeling and analysis for mobile application services: The perspective of mobile network operators. 111, pp. 146-163.

Wang, Z., \& Kim, H. G. (August de 2017). Can Social Media Marketing Improve Customer Relationship Capabilities and Firm Performance? Dynamic Capability Perspective. Journal of Interactive Marketing, 39, pp. 15-26.

Wieneke, A., \& Lehrer, C. (August de 2016). Generating and exploiting customer insights from social media data. Electronic Markets, 26(3), pp. 245-268.

Wilden, R., \& Gudergan, S. P. (March de 2015). The impact of dynamic capabilities on operational marketing and technological capabilities: investigating the role of environmental turbulence. Journal of the Academy of Marketing Science, 43(2), pp. 181-199.

Yoo, Y., Henfridsson, O., \& Lyytinen, K. (November de 2010). Research Commentary Long Tails vs. Superstars: The Effect of Information Technology on Product Variety and Sales Concentration Patterns. Information Systems Research, 21(4), pp. 736-747.

Yun, Q., \& Gloor, P. A. (December de 2015). The web mirrors value in the real world: comparing a firm's valuation with its web network position. Computational and Mathematical Organization Theory, 21(4), pp. 356-379.

Zavattaro, S. M., \& Daspit, J. J. (January de 2016). A grounded theoretical approach to understanding innovation in destination marketing organizations. Journal of Vacation Marketing, pp. 1-16.

Zavattaro, S. M., Daspit, J. J., \& Adams, F. G. (April de 2015). Assessing managerial methods for evaluating place brand equity: A qualitative investigation. Tourism Management, 47, pp. 11-21.

Zollo, M.; Winter, S. (2002). Deliberate learning and the evolution of dynamic capabilities. Organization Science, 13(3), 339-351.

The Association of Business Schools (ABS). (2015). Academic Journal Guide 2015. Londres. Recuperado de http://gsom.spbu.ru/files/abs-list-2015.pdf

Whetten, D. A. (1989). What constitutes a theoretical contribution? The Academy of Management Review, 14(4), p. 490-495. 